\section{Experimental Stabilization of Chaos in a Voltage-Mode DC Drive System}

\author{
J. H. Chen, K. T. Chau, S. M. Siu, and C. C. Chan
}

\begin{abstract}
This paper first presents experimental evidence on the use of delayed self-controlling feedback to stabilize chaos in a practical voltage-mode de drive system. Also, a new analytical approach to compute the domain of stabilization is proposed. Based on a simple feedback loop, chaotic behavior can be successfully stabilized to fundamental or subharmonic operation using the same time delay.
\end{abstract}

Index Terms-Bifurcation, chaos, dc motor drives, delay effects, stability, voltage control.

\section{INTRODUCTION}

In the past decades, chaos was misinterpreted in industrial practice: it was deemed a noise when the bounded range of chaotic behavior was narrow, whereas it was classified as an unstable phenomenon when the bounded range was wide. Starting from the late 1980's, chaos has been identified to be a real phenomenon in power electronics. Then, many investigations into chaotic behavior of dc-dc converters were conducted in the 1990's. In 1999, the investigation was extended to industrial dc drive systems [1].

Control of chaos has received much attention in recent years. In [2], an approach was proposed in which the feedback control signal is generated by the difference between the current state of the system and the corresponding state delayed by one period of the desired periodic orbit. This delayed self-controlling feedback approach and its variants have the advantage that the only information needed about the desired orbit is its period and no intensive computation is necessary to generate the control signal. Recently, this approach has been theoretically applied to stabilize chaotic behavior of a dc-dc buck converter [3].

The purpose of this paper is first to apply the delayed self-controlling feedback to stabilize chaos in an industrial power electronic system, namely, the voltage-mode dc drive. Different from [2] and [3], chaotic behavior will be stabilized to fundamental (period 1) or subharmonic (period $p$ ) operation using the same time delay. Moreover, a new analytical approach to compute the domain of stabilization will be proposed. Finally, theoretical derivation will be supported by both computer simulation and experiment measurement.

\section{DC DRIVE SYSTEM}

A schematic of the experimental dc drive system is shown in Fig. 1. In principle, there are three main subsystems, namely, a power electronic dc chopper, a motor-generator set, and an analog electronic controller. The dc chopper, consisting of a dc power supply $V_{s}$, a power MOSFET switch IRFI640G, a power diode BYW29E200, and an inductor $L$, functions to regulate the input power flowing into the drive system. The value of $L$ is chosen in such a way that the armature current $i$ of the motor is always the positive so-called continuous conduction mode of operation. The motor generator set includes a dc motor, a dc generator, a coupler, and an electronic load, where the mechanical load

Manuscript received June 20, 1999; revised November 12, 1999. This work was supported in part by a grant from the Research Grants Council of Hong Kong Special Administrative Region, China, under Project HKU 7128/99E. This paper was recommended by Associate Editor C. W. Wu.

The authors are with the Department of Electrical and Electronic Engineering, University of Hong Kong, Pokfulam, Hong Kong, China.

Publisher Item Identifier S 1057-7122(00)05500-8. torque $T_{l}$ is electronically controlled by the current sink of the electronic load. The electronic controller involves simple hardware, namely an encoder M57962L, a frequency-to-voltage (F/V) converter LM331, three op amps (OA1, OA2, and OA3) LM833, a bucket-brigade delay (BBD) line MN3004 and its clock MN3101, a ramp-signal generator, a comparator (CM) LM311, and a MOSFET driver DS0026. Based on the encoder and an F/V converter, the motor speed is converted into an analogue signal $v_{\omega}$ with gain $\gamma$ ), which is then compared with the command speed signal $v_{\omega}^{*}$ to produce the error signal $v_{e}$ via OA 1 with gain $\alpha$. Hence, the speed feedback gain $g$ equals $\alpha \gamma$. According to the principle of delayed self-controlling feedback [2], $v_{e}$ and its delayed version $v_{\tau}=v_{e}(t-\tau)$ are fed into OA2 with gain $\beta$ to produce the perturbation signal $v_{p}$, which is then compared with $v_{e}$ to generate the desired control signal $v_{c}$ via OA3 with gain unity. Finally, $v_{c}$ is compared with the ramp signal $v_{r}$ (with period $T$ and upper and lower bound voltages $v_{u}$ and $v_{l}$ ) via CM to produce the PWM switching signal for driving the power MOSFET. The core of this controller is the BBD line and the associated clock. By tuning the clock frequency via its externally connected $R_{1}-R_{2}-C$ network, the BBD line can allow for a time delay varying from 2.56 to $25.6 \mathrm{~ms}$. In the controller, the time delay $\tau$ is set to the switching period $T$ which, in fact, corresponds to fundamental operation (the period- 1 orbit).

The dynamics of the dc drive system using delayed self-controlling feedback can be described by a Poincaré map $P: R^{2} \rightarrow R^{2}$. This map is defined as an iterative function that maps the state vector solution $\mathbf{X}(t)$ at $t=n T$ to its successive one at $t=(n+1) T: \mathbf{X}_{n+1}=$ $P\left(\mathbf{X}_{n}\right)$. Thus, the fundamental solution corresponds to a fixed point $\mathbf{X}^{*}$ of the Poincaré map is given by

$$
\begin{aligned}
\mathbf{X}^{*}= & -\mathbf{A}^{-1} \mathbf{E}_{1}+(\mathbf{1}-\Phi(T))^{-1} \\
& \cdot\left(\mathbf{1}-\Phi\left(T-\delta_{1} T\right)\right) \mathbf{A}^{-1}\left(\mathbf{E}_{1}-\mathbf{E}_{2}\right) \\
h\left(\delta_{1}\right)= & \mathbf{E}_{3}\left[-\mathbf{A}^{-1} \mathbf{E}_{1}+\Phi\left(\delta_{1} T\right)(\mathbf{1}-\Phi(T))^{-1}\right. \\
& \left.\cdot\left(\mathbf{1}-\Phi\left(T-\delta_{1} T\right)\right) \mathbf{A}^{-1}\left(\mathbf{E}_{1}-\mathbf{E}_{2}\right)-\mathbf{E}_{4}\right] \\
& -v_{r}\left(\delta_{1} T\right)=0
\end{aligned}
$$

where $\mathbf{X}=(\omega, i), \Phi(t)=\exp (\mathbf{A} t), \delta_{1}$ is the duty cycle and $\mathbf{A}, \mathbf{E}_{1}, \mathbf{E}_{2}, \mathbf{E}_{3}, \mathbf{E}_{4}$ are matrices depending on the system parameters. The fixed point is stable if and only if the eigenvalues of its Jacobian matrix $\mathbf{J}_{1}=D P\left(\mathbf{X}^{*}\right)$ all lie within the unit circle in the complex plane. This matrix is given by

$$
\mathbf{J}_{1}=\Phi\left(T-\delta_{1} T\right)(\mathbf{1}+(1-\beta) \mathbf{C}+\beta \mathbf{C} \Phi(-T)) \Phi\left(\delta_{1} T\right)
$$

where $\mathbf{C}$ is a matrix dependent on $\mathbf{X}^{*}, \delta_{1}, \mathbf{A}, \mathbf{E}_{1}, \mathbf{E}_{2}, \mathbf{E}_{3}$ and $\mathbf{E}_{4}$. It indicates that the period-1 orbit can be stabilized by selecting a suitable $\beta$ using (1) and (2).

The approach to compute the domain of stabilization for the period- $p$ ( $p>1$ ) orbit using $\tau=T$ is exemplified by the period-2 case. Considering $\delta_{1} \geq \delta_{2}$, the corresponding period-2 orbit $\left(\mathbf{X}_{1}^{*}, \mathbf{X}_{2}^{*}\right)$ and Jacobian matrix $\mathbf{J}_{2}=D P^{(2)}\left(\mathbf{X}_{1}^{*}\right)$ are given by

$$
\begin{aligned}
\mathbf{X}_{1}^{*}= & \mathbf{A}^{-1} \mathbf{E}_{1}+(\mathbf{1}-\Phi(2 T))^{-1}\left[\Phi(T)\left(\mathbf{1}-\Phi\left(T-\delta_{1} T\right)\right)\right. \\
& \left.+\left(\mathbf{1}-\Phi\left(T-\delta_{2} T\right)\right)\right] \mathbf{A}^{-1}\left(\mathbf{E}_{1}-\mathbf{E}_{2}\right) \\
\mathbf{X}_{2}^{*}= & -\mathbf{A}^{-1} \mathbf{E}_{1}+(\mathbf{1}-\Phi(2 T))^{-1}\left[\left(\mathbf{1}-\Phi\left(T-\delta_{1} T\right)\right)\right. \\
& \left.+\Phi(T)\left(\mathbf{1}-\Phi\left(T-\delta_{2} T\right)\right)\right] \mathbf{A}^{-1}\left(\mathbf{E}_{1}-\mathbf{E}_{2}\right)
\end{aligned}
$$




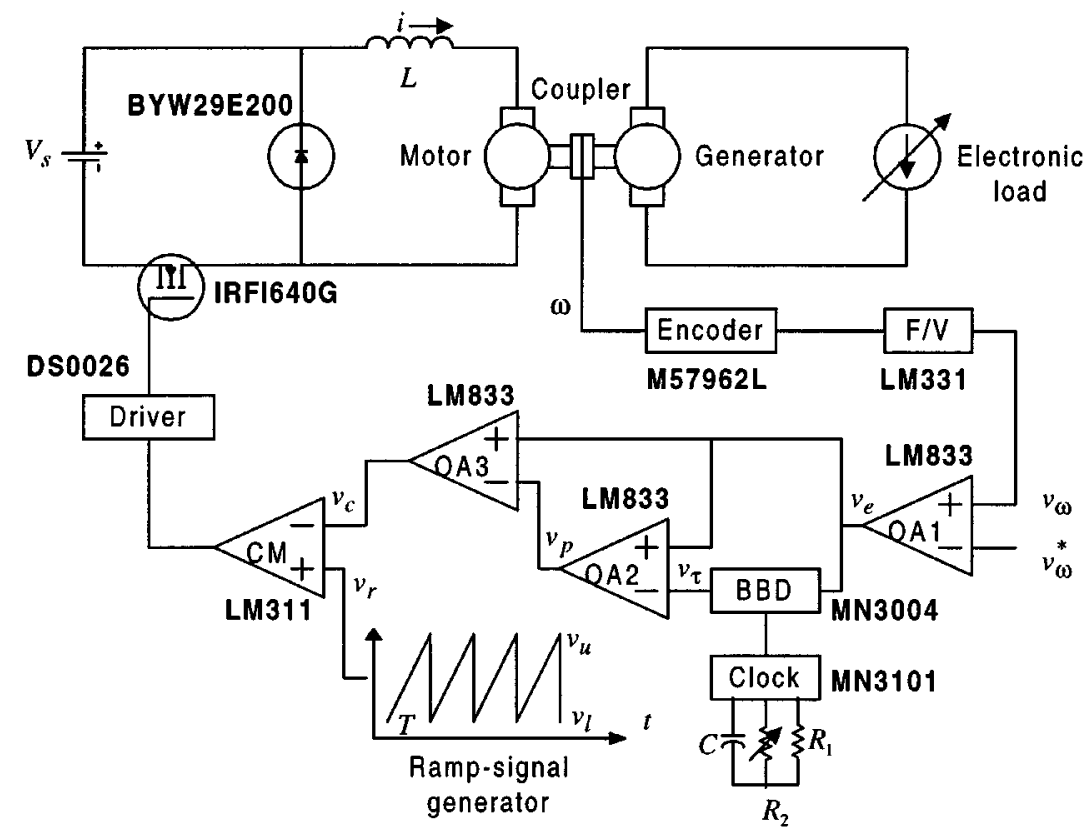

Fig. 1. Schematic of experimental setup.

$$
\begin{aligned}
h_{1}\left(\delta_{1}, \delta_{2}\right)= & \mathbf{E}_{3}\left[-\mathbf{A}^{-1} \mathbf{E}_{1}+\Phi\left(\delta_{1} T\right)\right. \\
& \cdot(\mathbf{1}-\beta(\mathbf{1}-\Phi(-T)))\left(\mathbf{X}_{1}^{*}+\mathbf{A}^{-1} \mathbf{E}_{1}\right) \\
& \left.+\beta\left(\mathbf{1}-\Phi\left(\delta_{1} T-T\right)\right) \mathbf{A}^{-1}\left(\mathbf{E}_{1}-\mathbf{E}_{2}\right)-\mathbf{E}_{4}\right] \\
& -v_{r}\left(\delta_{1} T\right)=0 \\
h_{2}\left(\delta_{1}, \delta_{2}\right)= & \mathbf{E}_{3}\left[-\mathbf{A}^{-1} \mathbf{E}_{1}+\Phi\left(\delta_{2} T\right)\left(\mathbf{X}_{2}^{*}+\mathbf{A}^{-1} \mathbf{E}_{1}\right)\right. \\
& \left.-\beta \Phi\left(\delta_{2} T\right)\left(\mathbf{X}_{2}^{*}-\mathbf{X}_{1}^{*}\right)-\mathbf{E}_{4}\right]-v_{r}\left(\delta_{2} T\right)=0 \\
\mathbf{J}_{2}= & \Phi\left(T-\delta_{2} T\right)\left(\mathbf{1}+(1-\beta) \mathbf{C}_{2}\right) \Phi\left(\delta_{2} T\right) \Phi\left(T-\delta_{1} T\right) \\
& \cdot\left(\mathbf{1}+(1-\beta) \mathbf{C}_{1}+\beta \mathbf{C}_{1} \Phi(-T)\right) \Phi\left(\delta_{1} T\right) \\
& +\Phi\left(T-\delta_{2} T\right)\left(\mathbf{1}+\beta \mathbf{C}_{2}\right) \Phi\left(\delta_{2} T\right)
\end{aligned}
$$

where $\mathbf{C}_{1}$ and $\mathbf{C}_{2}$ are matrices dependent on $\left(\mathbf{X}_{1}^{*}, \mathbf{X}_{2}^{*}\right),\left(\delta_{1}, \delta_{2}\right)$, $\mathbf{A}, \mathbf{E}_{1}, \mathbf{E}_{2}, \mathbf{E}_{3}$ and $\mathbf{E}_{4}$. Hence, the domain of stabilization for the period-2 orbit can be determined by using (3)-(5).

\section{RESULTS}

The default parameters of the experimental dc drive system are $V_{s}=$ $60 \mathrm{~V}, L=25 \mathrm{mH}, T_{l}=0.5 \mathrm{Nm}, v_{u}=2.2 \mathrm{~V}, v_{l}=0 \mathrm{~V}, T=6.667 \mathrm{~ms}$, $v_{\omega}^{*}=7 \mathrm{~V}$ (which corresponds to $105 \mathrm{rads}^{-1}$ ), and $g=1.4 \mathrm{~V} / \mathrm{rads}^{-1}$. The value of $g$ or $V_{s}$ is selected as the variable to create chaotic operation (called the bifurcation parameter) while the other parameters are fixed. Based on (1)-(5), the domain of stabilization for both bifurcation parameters $g$ and $V_{s}$ are shown in Fig. 2, in which the regions $D_{1}$ and $D_{2}$ stand for the domains for successful period-1 and period-2 stabilization, respectively.

In order to illustrate the stabilization of chaos, the system initially operates in chaos without employing the delayed self-controlling feedback (simply setting $\beta$ to zero). When $g$ is $1.6 \mathrm{~V} / \mathrm{rads}^{-1}$, the resulting chaotic behavior can be described by two state variables, namely, $i$ and $v_{c}$. Fig. 3(a) shows the simulated chaotic phase portrait as characterized by a random-like but bounded trajectory whose boundaries are $v_{c}$ $\in[0 \mathrm{~V}, 3.3 \mathrm{~V}]$ and $i \in[1.3 \mathrm{~A}, 6.8 \mathrm{~A}]$. As shown in Fig. 3(b) and (c), the chaos can be stabilized to the period- 1 and period- 2 orbit when $\beta$ is set to 0.15 and 0.1 , respectively. The corresponding boundaries are $v_{c}$

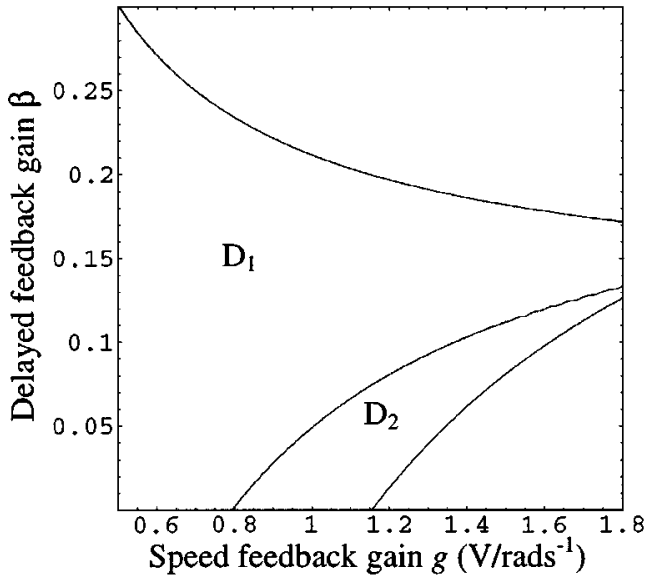

(a)

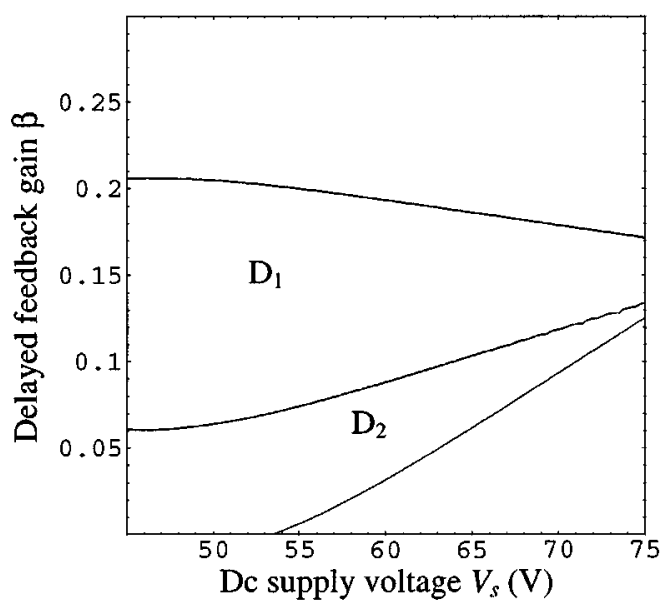

(b)

Fig. 2. Domain of stabilization. (a) Delayed feedback gain versus speed feedback gain. (b) Delayed feedback gain versus dc supply voltage. 


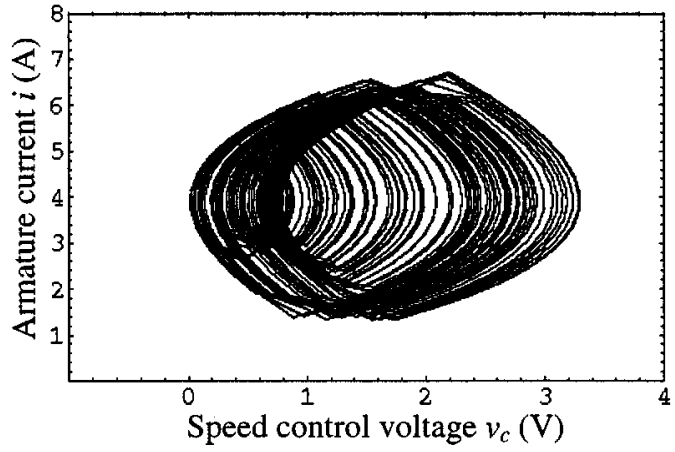

(a)

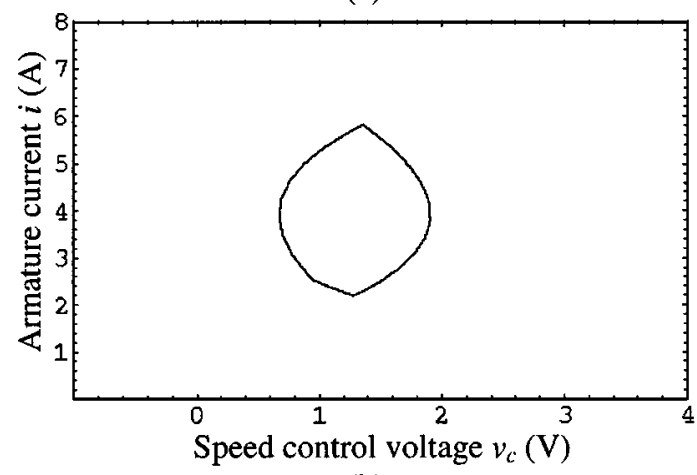

(b)

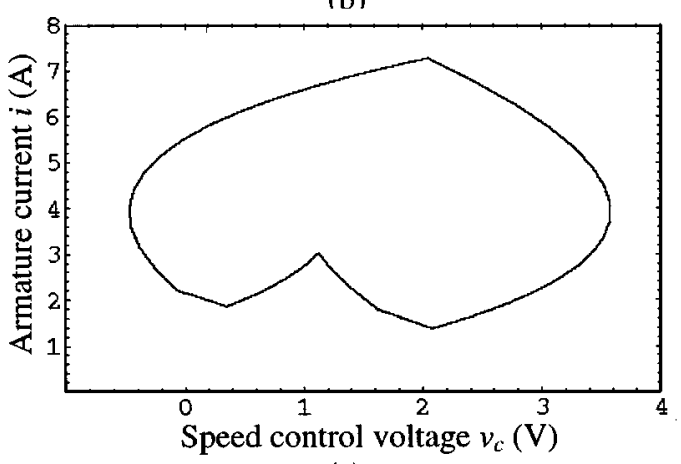

(c)

Fig. 3. Simulated phase portraits. (a) Chaotic orbit. (b) Period-1 orbit. (c) Period-2 orbit.

$\in[0.66 \mathrm{~V}, 1.9 \mathrm{~V}]$ and $i \in[2.2 \mathrm{~A}, 5.8 \mathrm{~A}]$ for the period-1 orbit, whereas $v_{c} \in[-0.5 \mathrm{~V}, 3.6 \mathrm{~V}]$ and $i \in[1.4 \mathrm{~A}, 7.4 \mathrm{~A}]$ for the period-2 orbit.

Based on experiments, the measured chaotic period-1 and period-2 phase portraits are shown in Fig. 4 . It can be found that $v_{c}$ $\in[-0.3 \mathrm{~V}, 3.5 \mathrm{~V}]$ and $i \in[1.1 \mathrm{~A}, 7.2 \mathrm{~A}]$ are the boundaries for the chaotic orbit, $v_{c} \in[-0.5 \mathrm{~V}, 2 \mathrm{~V}]$ and $i \in[2.1 \mathrm{~A}, 6.7 \mathrm{~A}]$ for the period-1 orbit, whereas $v_{c} \in[-1 V, 4 V]$ and $i \in[1.1 A, 7.5 A]$ for the period-2 orbit. Comparing Figs. 3 and 4, the simulated and measured results have a good agreement. Nevertheless, the boundaries of those phase portraits still have some discrepancies and the stabilized orbits are slightly shaking, which is due to some inevitable imperfections of the dc drive system, such as the uneven contacts of the dc commutator, the torsional oscillation of the coupler and the phase distortion of the BBD line.

\section{CONCLUSION}

In this paper, the experimental stabilization of chaos in a practical voltage-mode dc drive system is presented. Based on the implementation of delayed self-controlling feedback, the chaos occurring at high values of $g$ or $V_{s}$ can be stabilized to the fundamental period-1 and sub-

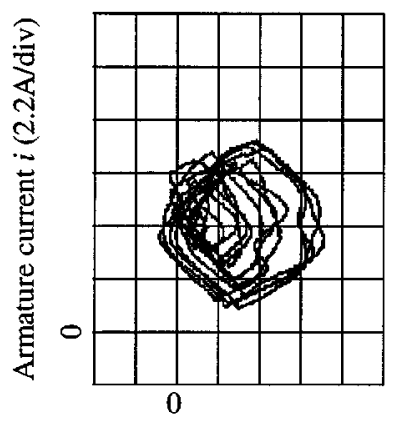

Speed control voltage $v_{c}(1 \mathrm{~V} /$ div $)$

(a)

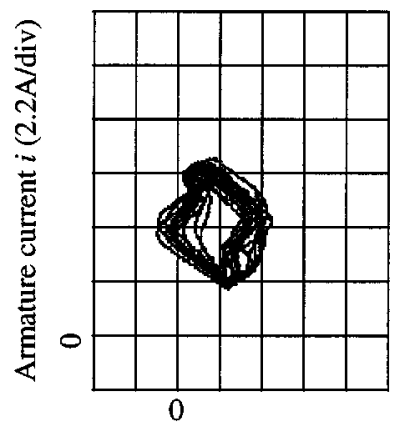

Speed control voltage $v_{c}$ (1V/div)

(b)

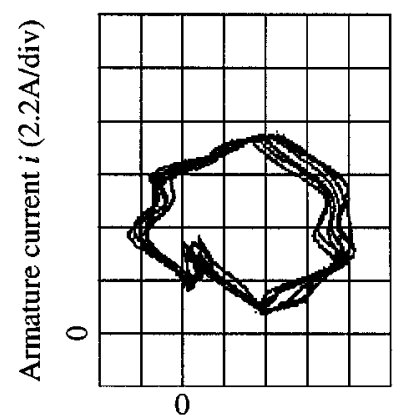

Speed control voltage $v_{c}(1 \mathrm{~V} / \mathrm{div})$

(c)

Fig. 4. Measured phase portraits. (a) Chaotic orbit. (b) Period-1 orbit. (c) Period-2 orbit.

harmonic period- $p$ orbits by tuning various values of $\beta$, while keeping $\tau$ constant. Moreover, a new analytical approach to compute the domain of stabilization is proposed. Since the approach is so general, it can readily be extended to stabilize the chaos due to other bifurcation parameters, such as $T_{l}, v_{u}, v_{l}$ and $T$.

\section{REFERENCES}

[1] J. H. Chen, K. T. Chau, and C. C. Chan, "Chaos in voltage-mode controlled DC drive systems," Int. J. Electron., vol. 86, no. 7, pp. 857-874, 1999.

[2] K. Pyragas, "Continuous control of chaos by self-controlling feedback," Phys. Lett. A, vol. 170, no. 6, pp. 421-428, 1992.

[3] C. Batlle, E. Fossas, and G. Olivar, "Time-delay stabilization of buck converter," in Proc. IEEE Int. Conf. Control Oscillation Chaos, 1997, pp. 590-593. 